\title{
La coexistence des générations en milieu urbain au Burkina Faso : Entre logique de sélection et obligation de parenté
}

\author{
Abdoulaye Maïga*1 2 and Banza Baya 23 \\ ${ }^{1}$ Université catholique de Louvain \\ Louvain-la-Neuve, Belgique \\ 2 Institut Supérieur des Sciences de la Population \\ Ouagadougou, Burkina Faso \\ ${ }^{3}$ Institut National de la Statistique et de la Démographie \\ Ouagadougou, Burkina Faso
}

\begin{abstract}
In Africa, household are traditionally characterized by residential arrangements with several generations cohabiting. This is partly linked to economical production systems and social reproduction scheme governed by a tacit intergenerational agreement. Due to modernization and urbanization, traditional patterns are differently affected resulting in a diffusion of modern standards of nuclear family. Given the plurality and complexity of urban family residential arrangements, this study aims to focus on the case of urban families arrangements in Burkina Faso and to analyse possible changes of residential arrangements in this country. The analysis models highlight an urban intergenerational residential patterns characterized by a duality based on a kind of reconciliation between traditional extended kinship and adapting to new social requirements of modernization resulting in a preference for a small size of family.
\end{abstract}

Keywords: Generations; Residential arrangements; Kinship; U rban area; Burkina Faso

\section{Résumé}

Les structures familiales étendues en Afrique sont bien souvent liées aux enjeux traditionnels de production et de reproduction sociale régis par un contrat intergénérationnel tacite. Avec la modernisation et l'urbanisation, les schèmes traditionnels sont variablement affectés, avec pour conséquence une recomposition des structures familiales suivant une logique individuelle ou de famille nucléaire au détriment du groupe familial étendu. Face à la pluralité et à la complexité des structures familiales urbaines, cette étude s'intéresse à la situation des familles urbaines burkinabés et les recompositions éventuelles de celles-ci, eu égard à la forte croissance urbaine qui caractérise le pays. Les modèles d'analyses descriptives et explicatives mettent en évidence des schémas familiaux urbains se caractérisant par une dualité reposant sur une sorte de conciliation entre les traditions familiales de parenté étendue et l'adaptation à de nouvelles exigences de modernisation se traduisant par une préférence voire une primauté de la famille restreinte.

Mots clé: Générations; Cohabitation; Parenté; Milieu urbain; Burkina Faso

\footnotetext{
* Abdoulaye Maïga

Centre de Recherche en Démographie et Sociétés,

Université catholique de Louvain,

Place Montesquieu 1, Boîte L2.08.03, Louvain-la-Neuve, 1348, Belgique

Tél : +3210478669

E-mail : mailayebf@yahoo.fr
} 


\section{Introduction}

Structure médiane entre l'individu et la société, la famille constitue le lieu par excellence de la production et de la reproduction biologique, sociale, culturelle et économique. L'étude des structures familiales africaines a focalisé bien d'attentions et d'intérêts scientifiques (Tabutin et Bartiaux 1986; Cordell et Piché 1987; Locoh 1988ab, 1997). II ressort globalement des études que la famille africaine traditionnelle se caractérise par une structure étendue complexe dans laquelle se mêlent des individus de différentes générations, apparentés ou non, liés par des liens de sang ou de mariage. Les structures familiales ont davantage été analysées sous un angle quantitatif global -familles élargies, nucléaires, polygames etc.- et moins dans une perspective intergénérationnelle. A ce propos, on note une émergence relativement récente d'études sur les relations intergénérationnelles (Antoine 2007 ; Vignikin 2007). Par rapport à la dimension résidentielle, il faut noter que les approches d'analyse tendent le plus souvent à privilégier le partage d'un même toit et des repas, la mise en commun des ressources, la reconnaissance de l'autorité d'une même personne comme chef pour la définition du concept de ménage. Cette définition fait abstraction du lien de parenté. Pour analyser les arrangements résidentiels intergénérationnels suivant les liens de sang ou de mariage des personnes, cette étude essaie de concilier les aspects traditionnellement pris en compte pour la définition du ménage et les liens de parenté des membres qui le composent.

Traditionnellement, les ménages africains se caractérisent par la cohabitation de plusieurs générations composés de grands parents, de parents, d'enfants et/ou de petits enfants. Avec la modernisation et la perception de la famille élargie comme un obstacle à la mobilité sociale, les modèles familiaux classiques s'estompent au profit de noyaux familiaux moins étendus en termes de membres et de générations (Ela 1992; Benga 1998). La désaffection pour les structures familiales larges au profit de choix plus individuels ou individualistes caractérisés par la coexistence d'un nombre plus limité de générations est un phénomène qui apparaît davantage comme l'apanage des centres urbains. Avec la forte croissance urbaine qu'enregistre le Burkina Faso, il y a donc lieu de s'interroger sur la tendance de cohabitation urbaine des générations dans ce pays. Les quatre recensements généraux de la population et de l'habitation (1975, 1985, 1996 et
2006) révèlent respectivement des taux d'urbanisation de $6,4 \%, 12,7 \%, 15,5 \%$ et $22,7 \%$ (IN SD 2000, 2008).

\section{Cadre théorique}

\section{La coexistence résidentielle sur fond de contrat intergénérationnel}

Contrôle familial par les ainés, solidarité lignagère ou devoirs de réciprocité de soutien des aînés par des cadets sont entre autres des valeurs et normes à la base de la constitution des modèles familiaux traditionnels en Afrique. L'organisation et le fonctionnement des sociétés traditionnelles africaines sont fortement marqués du sceau des vieilles personnes, garants et dépositaires des normes et valeurs sociales. Dans ces sociétés gérontocratiques, les ménages sont souvent constitués de plusieurs générations à la tête desquelles se trouvent généralement un patriarche qui centralise les ressources et détient le pouvoir de décision au sein de la famille ou du lignage. Les autres membres du ménage ont en principe obligation de regroupement autour du patriarche envers qui ils ont un devoir de respect et de vénération. En retour, celui-ci leur accorderait ses bénédictions, assurerait leur protection contre les maléfiques et organiserait leur mobilité sociale. La mobilité sociale fait référence aux possibilités de changements de statuts, de positions ou de fonctions des individus dans leurs sociétés ou familles d'appartenance. Elle peut être ascendante ou descendante et se manifester par une transition au niveau socioéconomique, un changement des prérogatives familiales des individus, ou l'accession à un statut social donné. Dans les sociétés traditionnelles, le moment et les modalités de la mobilité sociale des membres d'un clan familial donné sont bien souvent influencés par le patriarche dudit clan. Son quitus, son implication ou sa bénédiction sont ainsi parfois indispensables pour l'accession de tout membre du clan au mariage, à la propriété foncière ou à la migration. Dans certains contextes, les membres de la famille qui ont eu à former d'autres noyaux familiaux, après mariage, demeurent le plus souvent dans la grande résidence familiale du patriarche où ils contribuent à la production et à la mobilisation de ressources communes. Par ce contrôle, un contrat est ainsi établi entre les aînés détenteurs des ressources économiques, sociales ou religieuses et les cadets qui ont la force de production, mais dont la mobilité sociale procède souvent de la volonté des aînés. 
Dans les sociétés où les solidarités lignagères l'emportent sur les solidarités conjugales, l'organisation familiale préférentielle est celle des grandes unités domestiques (Ela 1992). Dans son acception classique, les ménages sont une constellation complexe d'individus de différentes générations liées par des liens de sang, de mariage ou non apparentés. Dans ces groupes élargis, cohabitent souvent ascendants, parents, proches descendants, descendants lointains et/ou collatéraux (Tabutin et Bartiaux 1986). La solidarité lignagère représente la logique à la base de ces constellations intergénérationnelles. Mais la logique de solidarité ne serait en fait qu'une logique de devoir ou de dettes réciproques entre générations (Marie 1997). Ce serait une sorte de contrat plus ou moins implicite entre générations qui se matérialise par la prise en charge, l'assistance et la solidarité réciproques entre ainés et cadets et principalement entre parents et enfants, à travers des échanges multiformes (Kinda 2006).

Au regard de ce pacte tacite, les parents auraient le devoir de donner à leurs enfants l'éducation, la protection, l'assistance nécessaire jusqu'à leur autonomie. C'est ainsi que dans certaines sociétés, l'échec des enfants est conçu comme une responsabilité des parents qui se doivent d'assumer la continuité de leur prise en charge (Roth 2007). De la naissance à leur affranchissement de la tutelle familiale, les enfants demeurent généralement sous le toit familial. Avec la scolarisation et le recul de l'âge au mariage, la cohabitation entre parents et enfants a tendance à se prolonger (Locoh 1998a). Durant leurs scolarités, les enfants quasiment dépendants des parents éprouvent généralement des difficultés pour la constitution de noyaux familiaux séparés. En outre, l'opportunité de fonder un propre ménage est peu envisagée lorsqu'ils ne sont pas encore mariés. Ce qui les conduit à vivre plus longtemps dans leur famille d'origine ou d'accueil. Cependant, le mariage ou l'entrée en vie féconde des enfants ne constitue pas toujours une condition nécessaire à la rupture du contrat de tutelle parentale. II n'est pas rare en effet d'enregistrer des ménages comprenant des parents, des enfants ainsi que des petits enfants.

De tels schémas de coexistence sont de plus courants avec la crise socioéconomique. Cette crise que vivent beaucoup de pays africains affecte de façon variable la rupture du cordon ombilical entre parents et enfants. "Une situation économique difficile peut [en effet] entraîner un écart croissant entre la demande des nouveaux noyaux familiaux et le parc de logements disponibles, ce qui conduit au maintien de familles étendues" (Locoh 1988a: 5.2.27). La coexistence des générations reflète, dans de tels cas de figure, des stratégies familiales pour permettre aux enfants de faire face à la crise. Avec l'accroissement de la population urbaine et l'incapacité du marché de l'emploi à absorber l'excédent démographique, les jeunes citadins sont de plus en plus exposés au chômage (Antoine et al. 1995). Ce qui influence négativement les possibilités d'autonomie financière et d'émancipation résidentielle. On observe par conséquent une prolongation de la durée de cohabitation entre parents et enfants, ainsi que le retard dans la constitution de nouvelles familles ou d'accession à l'autonomie par les enfants liés au contexte de crise économique, d'espace résidentiel limité, à la rareté et à la cherté des logements dans les villes (Antoine et al. 1995; Pilon et Vignikin 2006). Cette perception de dépendance des cadets vis-à-vis des aînés en situation de crise devrait cependant être relativisée, car "derrière l'apparente «dépendance résidentielle » des jeunes, se dessinent de nouvelles dépendances économiques des aînés contraints par leur perte de légitimité économique à partager l'autorité" (Diagne et Lessault 2007 : 35). Un tel transfert de l'assistance entre aînés et cadets s'inscrit dans les flux de réciprocité de la dette que régit le contrat intergénérationnel. Ce transfert de responsabilité et l'inversion des flux d'assistance des cadets vers les aînés s'inscrivent dans le principe de réciprocité de la dette sociale que régit le contrat intergénérationnel.

Dans la logique du contrat intergénérationnel, une assistance socioéconomique en retour des aînés par les cadets est une situation en principe escomptée. L'investissement consenti par les aînés envers les cadets constitue une sorte de dette que ces derniers se doivent d'acquitter lorsqu'ils auront atteint l'autonomie ou que les aînés seraient dans l'incapacité de pourvoir à leurs propres besoins durant la vieillesse. En réponse à cette dette, de nombreux enfants accueillent dans leurs foyers leurs parents durant leurs vieux jours. Dans ces sociétés, les enfants représenteraient une certaine assurance sociale vieillesse des parents (Kinda 2006). La réussite des enfants est appréhendée comme l'aboutissement des efforts des parents (Marie 1997). Ces derniers peuvent de ce fait estimer être en droit d'espérer une assistance réciproque de la 
part de leurs fils ou filles en les accueillant dans leurs foyers à l'âge de la vieillesse.

S'il est admis que les grands ménages, avec cohabitation de plusieurs générations, constituent les modèles familiaux par excellence (Baya et Laliberté 2008), il ne faut pas perdre de vue que la famille africaine vit présentement une certaine crise ou une transition avec la modernisation et l'urbanisation.

\section{La coexistence des générations à l'épreuve de la crise familiale}

A l'instar des populations et des phénomènes démographiques, les structures familiales ne sont ni statiques dans le temps ni similaires dans l'espace (Tabutin et Bartiaux 1986 ; Ela 1992). Elles évoluent et s'adaptent aux contextes, aux contraintes, aux influences de facteurs endogènes et exogènes. Avec la scolarisation, la modernisation ou l'urbanisation, les systèmes de valeurs communautaires cèdent progressivement le terrain à des normes et comportements plus individualistes. La primauté de la logique individuelle ou de couple sur la logique de parenté conduit à la recomposition sociale et familiale par la mise en place de ménages plus réduits et centrés sur la famille nucléaire (Ela 1992).

L'individualisation des modes de vie au détriment de la communauté tribale en Afrique s'inscrirait dans le processus normal de la théorie fonctionnaliste de Talcott Parsons (1955) cité par Vimard et N'cho (1997). Le postulat à la base de cette théorie est que la modernisation sociale, l'industrialisation et I'urbanisation conduisent à une distanciation des réseaux familiaux se traduisant par le passage de la famille étendue traditionnelle à la famille nucléaire moderne. En privilégiant la conjugalité ou la parentalité directe au détriment du groupe tribal ou communautaire, les nouveaux noyaux familiaux offrent des perspectives plus réduites de cohabitations de plusieurs générations. Dans un contexte urbain caractérisé par de nouvelles normes, de nouveaux comportements, de nouvelles aspirations, la famille élargie est appréhendée comme un obstacle à la mobilité sociale (Tabutin et Bartiaux, 1986 ; Benga, 1998). Les projets de vie sont dans ce cas conçus dans le sens de la promotion d'un nombre réduit de générations, généralement les parents et les enfants directs.

La tendance à la nucléarisation des ménages trouve en partie ses fondements dans la crise économique. L'effet de cette crise sur la constitution des modèles familiaux est ambivalent. Dans un sens, elle contribue à la mise en place de noyaux familiaux larges, à travers un réseau de solidarité, pour faire front à la dégradation des conditions de vie d'une partie du groupe familial ou lignager. Dans un autre sens, la crise économique est un facteur de décomposition et de réduction de la taille des ménages. La rareté des ressources crée une logique de sélection ou de filtrage des parents susceptibles de bénéficier de la solidarité résidentielle. Plutôt que d'accueillir leurs parents chez eux en ville, certains enfants prennent l'option de faire parvenir des subsides à leurs parents restés en milieu rural (Vignikin 2007).

Comme on peut le constater à partir de l'effet de la crise sur l'élargissement des ménages via la solidarité intergénérationnelle ou leur restriction du fait de l'effritement de la solidarité, les modèles familiaux en ville ne sont pas homogènes.

\section{La dualité résidentielle dans les ménages urbains}

Si la tendance récente est à la nucléarisation des ménages en ville, il faut néanmoins relever une certaine résistance, au vent de la modernisation, de la logique de parenté élargie, par concentration de générations et de collatéraux (Ela 1992; Marie 1997; Pilon et Vignikin 2006). Avec la crise économique, les effets des ajustements structurels, les exigences de la vie urbaine et les aspirations socioéconomiques plus individualisées, les stratégies d'adaptation des ménages urbains s'orientent de plus en plus vers des modèles familiaux plus réduits. Dans un autre sens, la crise a pour effet contraire de renforcer les liens familiaux dans un élan de solidarité et de soutien de la parentèle. $N$ onobstant l'émergence d'une logique individualiste, l'obligation de parenté et les valeurs de solidarité continuent dans certains contextes d'être des principes fondamentaux de la constitution de ménages avec plusieurs membres et de différentes générations. Contrairement à la perception classique et unilatérale de la migration urbaine comme un facteur de création de nouveaux ménages et donc d'accroissement des ménages isolés ou nucléaires, les traditions familiales et les habitudes culturelles conduisent le plus souvent les ménages citadins à accueillir un parent de la même famille, du même lignage ou originaire du même village (Locoh 1988a). $\mathrm{Du}$ fait du caractère relativement récent de l'urbanisation, la plupart des populations dans les villes ne s'y sont installées que depuis une, deux ou trois générations. II n'est par conséquent guère surprenant d'observer des modèles familiaux de type traditionnel élargis à côté de cellules familiales dans lesquelles la primauté de la parenté élargie est remise en cause (Ela 1992 ; Pilon et Vignikin 2006). En outre, on note une influence négative de I'ancienneté de l'urbanisation sur l'obligation de 
parenté, engendrant une distanciation des liens avec la parentèle étendue (Marie 1997).

Les contextes culturels, les contraintes socioéconomiques, les typologies de sociétés et le degré d'urbanisation influencent suivant des mécanismes variés les schémas familiaux et résidentiels. Avant d'analyser la situation des modèles familiaux dans les villes burkinabés, la prochaine section présentera l'approche méthodologie adoptée pour y parvenir.

\section{Données et méthodes}

Pour cette étude sur la coexistence des générations, une revue documentaire a permis de faire l'état de la situation des productions scientifiques relatives aux structures familiales et aux relations intergénérationnelles. Ce qui constitue une base théorique dans une perspective comparative des modèles de cohabitation intergénérationnelle du Burkina Faso et d'autres contextes.

Pour cerner la réalité empirique de la coexistence des générations au Burkina Faso, l'ensemble des quatre Enquêtes Démographiques et de Santé (EDS) ou Demographic and Health Surveys (DHS) réalisées au Burkina Faso ont servi de sources de données. II s'agit des DHS de 1993, de 1998-99, de 2003 et de 2010. A partir de ces bases de données, il a été possible de reconstituer les différentes générations, à travers la variable relative aux liens des membres du ménage avec le chef de ménage. La génération des grands-parents $(G 1)$, celle des parents (G2), celle des enfants $(G 3)$ et celles des petits enfants (G4) ont ainsi pu être reconstituées. Chaque membre d'un ménage a été identifié suivant chacune de ces quatre générations. Ce qui a permis de connaître l'existence d'une génération donnée dans un ménage et le nombre total de génération dans un ménage. A partir de la combinaison des différentes générations présentes dans chaque ménage, il a été possible de déterminer la structure des ménages en termes de générations, à travers un indicateur composite de générations. Les quatre types de générations se retrouvent à travers huit combinaisons de cohabitation intergénérationnelle dans les ménages (tableau 1 ). Un ménage peut comprendre une seule génération ou être constitué de la combinaison de deux, trois ou quatre générations.

Les variables explicatives de l'étude concernent le sexe du chef de ménage, le niveau d'instruction du chef de ménage, le statut matrimonial du chef de ménage, le niveau de vie du ménage, la présence dans le ménage de chacune des générations.
Comme variables de contrôle, on a l'âge du chef de ménage, le nombre de membres de ménage, le nombre d'enfants de moins de 15 ans dans le ménage. Les variables à expliquer sont la présence dans le ménage de la génération des grands-parents, la présence dans le ménage de la génération des enfants, et la présence dans le ménage de la génération des petits enfants. Pour l'ensemble des analyses, les données ont été préalablement pondérées pour tenir compte de l'effet d'échantillonnage et de stratification.

Après vérification de l'existence de relations statistiquement significatives entre les variables, des analyses descriptives et explicatives ont été conduites pour l'analyse de la coexistence des générations. L'approche descriptive a notamment consisté à des analyses tabulaires pour mettre en évidence la taille des ménages en termes de nombre d'individus et de générations. La part des différentes générations dans les ménages ainsi que la combinaison des générations dans les ménages ont aussi fait l'objet de ces analyses descriptives. Les analyses ont aussi cherché à mettre en évidence les évolutions de la structure des ménages sur la période de 1993 à 2010. L'analyse explicative, à travers des régressions logistiques, a permis de rechercher les facteurs explicatifs de la présence dans les ménages de la génération des grandsparents, de celle des enfants et enfin de celle des petits enfants.

Dans une perspective comparative et dans le souci de cerner les spécificités des relations intergénérationnelles urbaines ou l'effet possible de la modernisation, à travers les changements de valeurs et de comportements, sur la coexistence des générations en ville, les différentes analyses ont été effectuées en élaborant des modèles urbains et des modèles ruraux.

\section{Résultats}

\section{Les structures des ménages urbains burkinabés}

A l'instar d'autres sociétés africaines, les ménages burkinabés se caractérisent par un nombre relativement élevé de membres, malgré la baisse enregistrée au cours du temps (figure 1a). La taille médiane des ménages au niveau national est en effet passée de 9 membres à 7 membres entre 1993 et 2010. N éanmoins, les ménages urbains sont moins étendus que ceux du milieu rural. La taille des ménages et son évolution dans le temps en milieu rural sont similaires à ce qui est observé pour l'ensemble du pays. En milieu urbain par contre, la moitié des ménages comptait en 2010 un nombre de 
membres inférieur ou égal à 6 . Cette taille médiane urbaine résulte de la baisse de deux points de la taille observée en 1993. Même si les ménages urbains sont moins étendus que ceux du milieu rural, il n'en demeure pas moins que les tailles dans ces deux milieux sont relativement importantes (figure 1a).

Au-delà de la taille des ménages, l'intérêt de I'analyse se situe aussi dans la composition des ménages, en termes de présence des différentes générations en leur sein. A ce propos, il faut logiquement relever que les enfants constituent la génération quasiment présente dans les ménages des parents (figure 1c). Le niveau de présence de cette génération dans les ménages a très peu fluctué dans le temps. De même, la présence de la génération des enfants dans les ménages en milieu rural reste prépondérante sur celle du milieu urbain au cours du temps. Avec une proportion 4\% inférieure à celle du milieu rural, environ 9 ménages urbains sur 10 enregistrent la présence d'au moins une personne appartenant à la génération des enfants en 2010. La différence entre ces deux milieux se situait dans la même proportion en 1993. II faut bien évidemment prendre en considération la forte fécondité qui prévaut au Burkina Faso, notamment en milieu rural pour l'explication de la forte représentation de la génération des enfants dans les ménages. Au Burkina Faso en 1993, une femme pouvait avoir en moyenne 6,9 enfants au cours de sa vie génésique. L'indice synthétique de fécondité (ISF) est estimé à 6 enfants par femme en 2010. Pour cette dernière année, cet indice est de 3,9 enfants pour une citadine contre 6,7 enfants pour une femme vivant en milieu rural (IN SD et ICF International, 2012).
Concernant la présence d'autres générations dans les ménages - qu'on pourrait qualifier de générations éloignées ou étendues- il faut noter de façon globale une présence légèrement plus importante de la génération des petits enfants (figure 1d) comparativement à celle des grandsparents (figure 1b). Concernant les petits enfants, leur présence est manifestement plus importante dans les ménages urbains par rapport aux ménages ruraux sur toute la période d'observation de 1993 à 2010 (figure 1d). Mieux, les différences entre le milieu urbain et le milieu rural se sont accentuées, passant de $2 \%$ en 1993 à $10 \%$ en 2010 . II en ressort une plus grande propension à l'extension des ménages urbains vers la génération des descendants. Si la présence de la génération des grands-parents était quasi similaire au début des années 1990 entre les milieux urbain et rural, de légères évolutions font s'opérer au fil du temps (figure 1b). En effet, en 1998 et 2003, on note une légère primauté du milieu rural sur le milieu urbain concernant la présence des grands-parents dans les ménages. Cependant, la situation va s'inverser en 2010 avec environ $13 \%$ des ménages urbains qui enregistrent la présence d'au moins une personne de la génération des grands-parents contre près de $10 \%$ des ménages ruraux. Le niveau de présence de la génération des grands-parents en milieu urbain en 1993 est quasiment le même que celui de 2010. Des fluctuations à la baisse ont par contre été enregistrées entre ces deux années. 


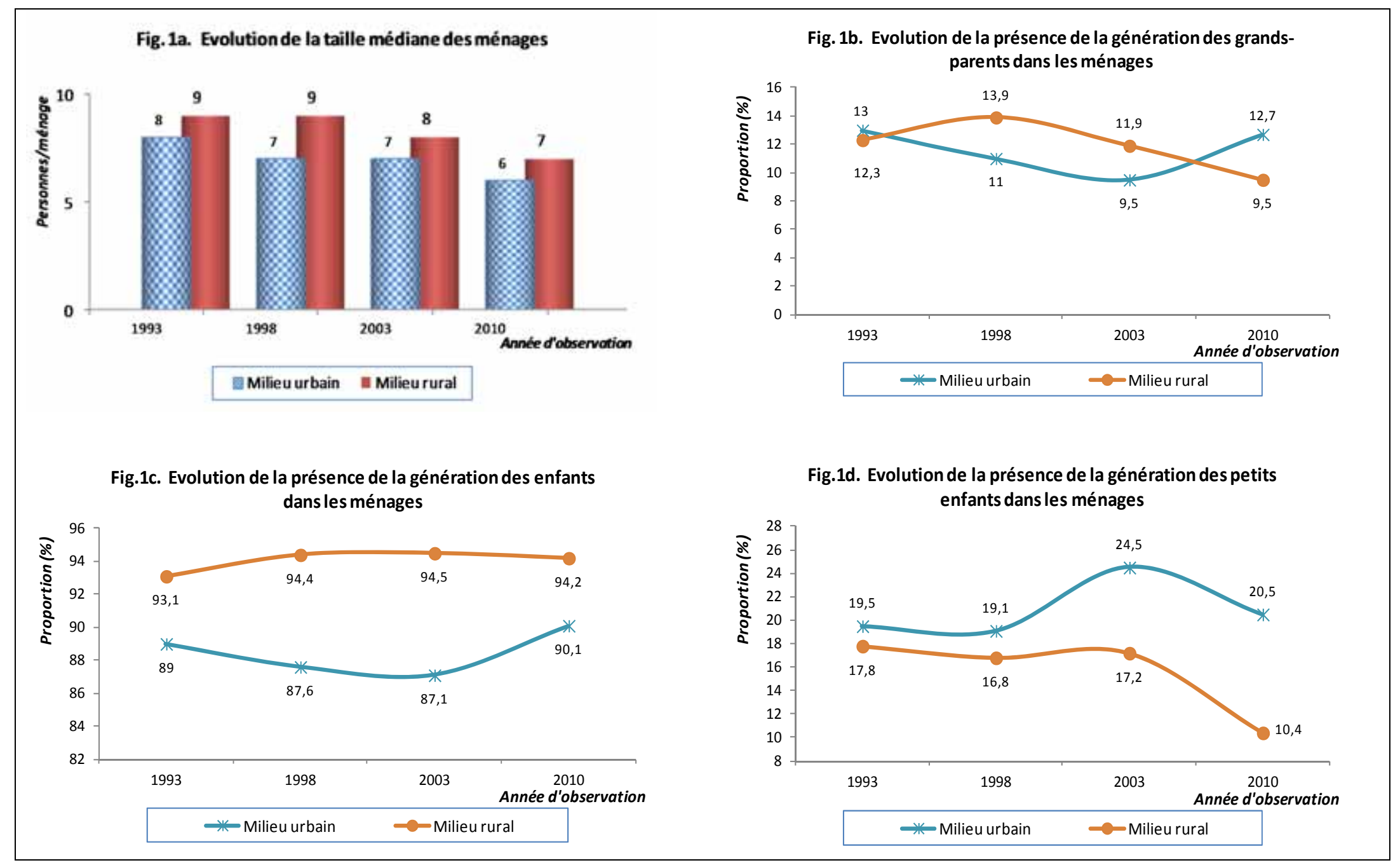

Figure 1. Evolution de la structure des ménages de 1993 à 2010 au Burkina Faso

Sources : Données Burkina Faso Demographic and Health Sunveys (DHS) 1993, 1998, 2003 et 2010 
L'analyse de la structure des ménages en fonction des générations qui les composent montre une prépondérance des ménages avec deux générations, essentiellement composées de générations de parents et d'enfants (tableau 1). La proportion de ménages composés uniquement des générations de parents et d'enfants est passée d'environ deux-tiers à près de trois-quarts entre 1993 et 2010. Cette évolution pour l'ensemble du pays est essentiellement liée à l'évolution d'environ $10 \%$ en milieu rural de cette catégorie de ménage au cours de la même période. Deux autres formes de ménages urbains avec deux générations existent, mais dans de faibles proportions. II s'agit de ménages comprenant les générations des grands-parents et des parents $(1,3 \%)$ et de ménages composés de parents et petits fils/filles $(1,4 \%)$ dont les proportions n'ont pratiquement pas varié entre 1993 et 2010. II en est de même pour ces deux structures de ménages en milieu rural. Dans la catégorie des ménages avec trois générations, c'est la combinaison "parents, enfants et petits enfants" qui est la plus importante. Cette combinaison de générations est deux fois plus importante en milieu urbain $(17,6 \%)$ qu'en milieu rural (8,5\%). La présence de ménages composés de "grands-parents, parents et enfants" a très peu évolué au cours du temps et est relativement moins importante $(9,7 \%$ en milieu urbain et $8,5 \%$ en milieu rural). Les ménages avec "grands-parents, parents et petits enfants" sont quant à eux assez rares. Si en nombre d'individus les ménages urbains sont moins grands que les ménages ruraux, il n'en est pas de même pour le nombre de générations qui les composent. Dans une proportion certes faible, la concentration des quatre générations - c'est-à-dire la présence à la fois des grands-parents, des parents, des enfants et des petits enfants- est plus importante dans les ménages urbains $(1,6 \%)$ que dans les ménages ruraux $(0,6 \%)$. Cette configuration n'a pratiquement pas évolué au cours du temps entre ces deux milieux de résidence. 
Tableau 1. Structuration des ménages en fonction de la présence des différentes générations

\begin{tabular}{|c|c|c|c|c|c|}
\hline \multirow[b]{2}{*}{ Milieu de résidence } & \multirow[b]{2}{*}{ Générations } & \multicolumn{2}{|l|}{1993} & \multicolumn{2}{|l|}{2010} \\
\hline & & $\begin{array}{l}\text { Proportion } \\
\text { (\%) }\end{array}$ & $\begin{array}{l}\text { Effectifs } \\
\text { pondérés }\end{array}$ & $\begin{array}{l}\text { Proportion } \\
(\%)\end{array}$ & $\begin{array}{l}\text { Effectifs } \\
\text { pondérés }\end{array}$ \\
\hline \multirow{9}{*}{$\begin{array}{l}\text { Urbain } \\
\text { Burkina Faso }\end{array}$} & G2 & 8,8 & 545 & 7,2 & 1319 \\
\hline & $\mathrm{G} 1+\mathrm{G} 2$ & 1,1 & 69 & 1,3 & 247 \\
\hline & $\mathrm{G} 2+\mathrm{G} 3$ & 60,7 & 3778 & 61,2 & 11240 \\
\hline & $\mathrm{G} 2+\mathrm{G} 4$ & 1,1 & 69 & 1,4 & 255 \\
\hline & $\mathrm{G} 1+\mathrm{G} 2+\mathrm{G} 3$ & 10,0 & 621 & 9,7 & 1789 \\
\hline & $\mathrm{G} 1+\mathrm{G} 2+\mathrm{G} 4$ & 0,0 & 0 & 0,0 & 4 \\
\hline & $\mathrm{G} 2+\mathrm{G} 3+\mathrm{G} 4$ & 16,4 & 1023 & 17,6 & 3227 \\
\hline & $\mathrm{G} 1+\mathrm{G} 2+\mathrm{G} 3+\mathrm{G} 4$ & 1,9 & 120 & 1,6 & 288 \\
\hline & Total & 100,0 & 6225 & 100,0 & 18369 \\
\hline \multirow{9}{*}{$\begin{array}{l}\text { Rural } \\
\text { Burkina Faso }\end{array}$} & $\mathrm{G} 2$ & 4,5 & 1298 & 3,7 & 2377 \\
\hline & $\mathrm{G} 1+\mathrm{G} 2$ & 0,8 & 241 & 0,6 & 395 \\
\hline & $\mathrm{G} 2+\mathrm{G} 3$ & 66,0 & 18891 & 76,7 & 49095 \\
\hline & $\mathrm{G} 2+\mathrm{G} 4$ & 1,5 & 429 & 1,5 & 951 \\
\hline & $\mathrm{G} 1+\mathrm{G} 2+\mathrm{G} 3$ & 10,8 & 3093 & 8,5 & 5452 \\
\hline & $\mathrm{G} 1+\mathrm{G} 2+\mathrm{G} 4$ & 0,0 & 0 & 0,0 & 14 \\
\hline & $G 2+G 3+G 4$ & 15,7 & 4489 & 8,5 & 5466 \\
\hline & $\mathrm{G} 1+\mathrm{G} 2+\mathrm{G} 3+\mathrm{G} 4$ & 0,6 & 177 & 0,4 & 241 \\
\hline & Total & 100,0 & 28617 & 100,0 & 63991 \\
\hline \multirow{9}{*}{$\begin{array}{l}\text { Ensemble du Burkina } \\
\text { Faso }\end{array}$} & G2 & 5,3 & 1842 & 4,5 & 3696 \\
\hline & $\mathrm{G} 1+\mathrm{G} 2$ & 0,9 & 310 & 0,8 & 642 \\
\hline & $\mathrm{G} 2+\mathrm{G} 3$ & 65,1 & 22669 & 73,3 & 60335 \\
\hline & $\mathrm{G} 2+\mathrm{G} 4$ & 1,4 & 498 & 1,5 & 1206 \\
\hline & $\mathrm{G} 1+\mathrm{G} 2+\mathrm{G} 3$ & 10,7 & 3714 & 8,8 & 7241 \\
\hline & $\mathrm{G} 1+\mathrm{G} 2+\mathrm{G} 4$ & 0,0 & 0 & 0,0 & 18 \\
\hline & $\mathrm{G} 2+\mathrm{G} 3+\mathrm{G} 4$ & 15,8 & 5512 & 10,6 & 8693 \\
\hline & $\mathrm{G} 1+\mathrm{G} 2+\mathrm{G} 3+\mathrm{G} 4$ & 0,9 & 297 & 0,6 & 528 \\
\hline & Total & 100,0 & 34842 & 100,0 & 82359 \\
\hline \multicolumn{6}{|c|}{ Légende } \\
\hline $\begin{array}{l}\mathrm{G} 1 \text { : Génération des } \mathrm{G} \\
\mathrm{G} 2 \text { : Génération des } \mathrm{Pa}\end{array}$ & parents & & $\begin{array}{l}\text { G3 : Génér } \\
\text { G4 : Génér }\end{array}$ & $\begin{array}{l}\text { des Enfants } \\
\text { des Petits enfa }\end{array}$ & \\
\hline
\end{tabular}

Sources : Données Burkina Faso Demographic and Health Surveys (DHS) 1993 et 2010

\section{Les facteurs associés de la présence des} différentes générations dans les ménages

Dans cette section, il s'agit d'une analyse des facteurs susceptibles d'expliquer la présence dans le ménage soit de la génération des grands-parents, soit de la génération des enfants ou de la génération des petits enfants. Pour chacune des générations, un modèle avec une composante urbaine et une composante rurale permet d'analyser les déterminants de leur présence respectivement dans les ménages urbains et dans les ménages ruraux.

L'analyse de la présence des générations dans le ménage suivant le sexe du chef de ménage révèle que les ménages dirigés par des hommes 
enregistrent une forte présence de la génération des grands-parents comparativement à ceux dirigés par les femmes. Autant en milieu urbain qu'en milieu rural, il y a $40 \%$ plus de chance de rencontrer la génération de grands-parents dans des ménages dont un homme est le chef que dans les ménages dont une femme en est responsable (tableau 2). II ressort que même si les grands-parents sont davantage présents dans les ménages des hommes que dans ceux des femmes, leur présence est similaire dans les deux milieux de résidence. La corrélation entre le niveau d'instruction et la présence de la génération des grands-parents dans le ménage ne s'est avérée statistiquement significative que pour le niveau d'instruction primaire en milieu rural et le niveau secondaire ou plus en milieu urbain. Comparativement aux chefs de ménage sans instruction, les chefs de ménages qui ont le niveau primaire sont $40 \%$ plus enclins à accueillir les grands-parents dans les ménages ruraux. Avec une corrélation statistiquement significative à $5 \%$, la probabilité d'accueil d'un parent de la génération des grands-parents par un chef de ménage de niveau d'instruction secondaire ou plus est supérieure de $10 \%$ par rapport à un chef de ménage sans niveau $d$ 'instruction en ville. Le statut matrimonial influence fortement l'accueil des grands-parents dans les centres urbains. La probabilité pour un chef de ménage marié d'accueillir des grands-parents en ville est près de deux fois supérieure à celle d'un chef de ménage célibataire. Cette probabilité est trois supérieure lorsque le chef de ménage à un statut de veuf ou de divorcé. Par contre, en milieu rural la génération des grands-parents a moins tendance à se retrouver dans les ménages dont le chef est marié. La situation en milieu rural laisserait ainsi entrevoir une sorte de rupture résidentielle des personnes avec leurs géniteurs une fois le statut matrimonial de marié acquis pour former leurs ménages à part. Autant en milieu urbain qu'en milieu rural, on note presqu'une corrélation négative entre le niveau de vie des ménages et l'accueil en leur sein d'une personne de la génération des grands-parents. Par rapport aux ménages de faible niveau de vie, les probabilités pour accueillir la vieille génération sont respectivement $10 \%$ et $20 \%$ inférieures pour les ménages de niveau de vie moyen et ceux de niveau de vie élevé. En ville, la probabilité de cohabitation des grands-parents dans un ménage de niveau de vie moyen est 30\% supérieure à celle d'un ménage de faible de niveau de vie. En revanche, les ménages urbains avec un niveau de vie élevé présentent une probabilité $30 \%$ inférieure à celle des ménages de faible niveau de vie concernant la présence d'une personne de la vieille génération. Les changements de systèmes de valeurs et de comportements sont des processus susceptibles d'affecter plus les populations urbaines généralement instruites et de niveau de vie élevé. Ce serait de ce fait une conséquence logique que la nucléarisation des ménages, consécutive à la modernisation, affecte davantage cette classe sociale. Autant dans les villes qu'en milieu rural, une présence de la génération des enfants ou de celle des petits enfants est corrélée à une plus faible présence de la génération des grands-parents dans les ménages.

Contrairement à la présence des grands-parents, la génération des enfants est davantage présente dans les ménages dont le chef est une femme que dans les ménages dirigés par un homme (tableau 2). En effet, la génération des enfants est 2 fois et 2,5 fois plus présente en milieu urbain et en milieu rural dans les ménages avec une femme pour chef de ménage. O n observe une réduction de la probabilité d'avoir une génération des enfants dans le ménage avec l'augmentation du niveau d'instruction de la génération des parents. En effet, les ménages urbains et ruraux avec un chef de niveau d'instruction secondaire ou plus ont respectivement $30 \%$ et $20 \%$ moins de chance d'avoir des enfants dans le ménage que les ménages du même milieu lorsque les chefs n'ont pas été à l'école. Ce constat est conforme à la corrélation négative généralement observée entre éducation des parents et fécondité (Tuman et al. 2007; Hugon 2008; Bongaarts 2010), dans la mesure où les populations instruites ont une fécondité moins élevée que les populations non instruites. Le fait que les chefs de ménages soient mariés ou veufs/divorcés influence fortement la présence des enfants dans les ménages autant dans les zones urbaines que dans les campagnes. Contrairement au statut matrimonial, le statut économique du ménage ne se révèle pas statistiquement significatif pour l'explication de la présence des enfants dans les ménages ruraux. En revanche dans les villes, la corrélation est statistiquement significative et positive. Les ménages avec un niveau de vie élevé ont près de deux fois plus de chance d'enregistrer la présence d'une personne appartenant à la génération des enfants que les ménages pauvres. La présence de la génération des grands-parents tout comme celle des petits-enfants sont négativement corrélées à la présence des enfants dans les ménages urbains et ruraux. 
A l'instar de la présence de la génération des enfants selon le sexe du chef de ménage, la génération de petits enfants est aussi et davantage présente dans les ménages dont le chef est une femme. En effet, la probabilité d'enregistrer la présence des petits enfants dans les ménages urbains est 10 fois plus grande dans les ménages des femmes que ceux des hommes. En milieu rural, cette probabilité a un ordre de grandeur de 5 dans le même sens. II ressort que selon le milieu de résidence, la génération des petits enfants est davantage présente dans les ménages urbains que ceux des villages. D'où la conclusion d'une plus grande présence de la génération des petits enfants dans les zones urbaines notamment dans les ménages dirigés par les femmes. II faut rappeler (tableau 1) que les petits enfants se retrouvent généralement dans des ménages composés de parents, d'enfants et de petits enfants. Ce qui laisse supposer que dans les ménages urbains et beaucoup plus dans ceux dirigés par des femmes, les enfants sont plus enclins à y rester plus longtemps et à y avoir une vie féconde, d'où la présence des petits enfants. Suivant le niveau d'instruction, on note une réduction de la présence des petits enfants pour les ménages urbains dont les responsables ont un niveau d'instruction élevé. A ce propos, il faut relever une présence de la génération des petits enfants 2 fois moins importante dans les ménages urbains dont le responsable à un niveau d'instruction correspondant au moins au secondaire. Par contre, en milieu rural la présence des petitsenfants est $50 \%$ plus importante dans les ménages quand les responsables ont un niveau d'instruction primaire que lorsqu'ils sont sans instruction. Concernant le statut matrimonial du chef de ménage, seuls les divorcés ou les veufs et uniquement dans les villes semblent avoir plus de personnes appartenant à la génération des petits enfants dans leurs ménages. Ainsi, la probabilité d'avoir un petit enfant au sein d'un ménage urbain est près de 2 fois supérieure lorsque dont le chef est veuf ou divorcé que lorsqu'il est célibataire. Tout comme pour la génération des enfants, le niveau de vie du ménage influence positivement la présence des petits enfants seulement dans les centres urbains. Dans les villes, les petits enfants sont en effet $80 \%$ et $60 \%$ davantage présents respectivement dans les ménages de niveau de vie moyen et de niveau de vie élevé par rapport aux ménages de faible niveau de vie. Aussi bien en milieu urbain qu'en milieu rural, la présence de la génération des grands-parents et de celle des enfants sont négativement corrélées à la présence des petits-enfants dans les ménages. 
Tableau 2. Déterminants de la présence dans les ménages de la génération des grands-parents, des enfants et des petits enfants (Modèle d'effets nets - Régression logistique - Odds ratio)

\begin{tabular}{|c|c|c|c|c|c|c|c|c|}
\hline \multirow[t]{2}{*}{ Variables } & \multicolumn{2}{|c|}{$\begin{array}{l}\text { Présence de génération de grands } \\
\text { parents dans le ménage }\end{array}$} & \multicolumn{4}{|c|}{$\begin{array}{c}\text { Présence de génération d'enfants } \\
\text { dans le ménage }\end{array}$} & \multicolumn{2}{|c|}{$\begin{array}{c}\text { Présence de génération de petits } \\
\text { enfants dans le ménage }\end{array}$} \\
\hline & Ménages urbains & Ménages ruraux & \multicolumn{2}{|c|}{ Ménages urbains } & \multicolumn{2}{|c|}{ Ménages ruraux } & Ménages urbains & Ménages ruraux \\
\hline \multicolumn{9}{|l|}{ 1. Sexe du chef de ménage } \\
\hline Féminin & 1,0 & 1,0 & 1,0 & & 1,0 & & 1,0 & 1,0 \\
\hline Masculin & $1,4 * * *$ & $1,4 * * *$ & 0,5 & $* * *$ & 0,4 & $* * *$ & $0,1 * * *$ & $0,2 * * *$ \\
\hline \multicolumn{9}{|l|}{ 2. Niveau d'instruction du chef de ménage } \\
\hline Sans instruction & 1,0 & 1,0 & 1,0 & & 1,0 & & 1,0 & 1,0 \\
\hline Primaire & $1,1 \mathrm{~ns}$ & $1,4 * * *$ & 0,9 & ns & 0,7 & $* * *$ & $1,2 * * *$ & $1,5 * *$ \\
\hline Secondaire & $1,1 * *$ & $1,0 \mathrm{~ns}$ & 0,7 & $* * *$ & 0,8 & $*$ & $0,5 * * *$ & $1,1 \mathrm{~ns}$ \\
\hline \multicolumn{9}{|l|}{ 3. Statut matrimonial chu chef de ménage } \\
\hline Célibataire & 1,0 & 1,0 & 1,0 & & 1,0 & & 1,0 & 1,0 \\
\hline Marié & $1,8 * * *$ & $0,3 * * *$ & 149 & $* * *$ & 1055 & $* * *$ & $0,9 \mathrm{~ns}$ & $0,8 \mathrm{~ns}$ \\
\hline Veuf/Divorcé & $3,4 * * *$ & $0,9 \mathrm{~ns}$ & 119 & $* * *$ & 959 & $* * *$ & $1,8 * *$ & $1,3 \mathrm{~ns}$ \\
\hline \multicolumn{9}{|l|}{ 4. Niveau de vie clu ménage } \\
\hline Faible & 1,0 & 1,0 & 1,0 & & 1,0 & & 1,0 & 1,0 \\
\hline Moyen & $1,3 * *$ & $0,9 * * *$ & 1,9 & *** & 1,1 & ns & $1,8 * * *$ & 1,0 ns \\
\hline Elevé & $0,7 * * *$ & $0,8 * * *$ & 1,9 & *** & 1,1 & $*$ & $1,6 * * *$ & $1,1 *$ \\
\hline \multicolumn{9}{|c|}{ 5. Présence de génération des grands parents clans le ménage } \\
\hline Non & & & 1,0 & & 1,0 & & 1,0 & 1,0 \\
\hline Oui & & & 0,2 & $* * *$ & 0,1 & $* * *$ & $0,5 * * * *$ & $0,6 * * *$ \\
\hline \multicolumn{9}{|c|}{ 6. Présence de génération des enfants clans le ménage } \\
\hline Non & 1,0 & 1,0 & & & & & 1,0 & 1,0 \\
\hline Oui & $0,8 * * *$ & $1,1 \mathrm{~ns}$ & & & & & $0,5 * * *$ & 0,4 *** \\
\hline \multicolumn{9}{|c|}{ 7. Présence de génération des petits enfants dans le ménage } \\
\hline Non & 1,0 & 1,0 & 1,0 & & 1,0 & & & \\
\hline Oui & $0,3 * * *$ & $0,3 * * *$ & 0,1 & $* * *$ & 0,05 & $* * *$ & & \\
\hline Significativité globale chu modèle & $* * *$ & $* * *$ & *** & & *** & & $* * *$ & $* * *$ \\
\hline Adéquation clu modèle $R^{2}$ & $11 \%$ & $16 \%$ & $53 \%$ & & $62 \%$ & & $46 \%$ & $28 \%$ \\
\hline Seuils de significativité : ns : non significatif & * : sic & gnificatif à $10 \%$ & & ** : signific & catif à 5\% & & $* * *$ : significatif & à $1 \%$ \\
\hline
\end{tabular}

Source : Données Burkina Faso Demographic and Health Sunvey (DHS) 2010 


\section{Discussions des résultats}

La pluralité des arrangements résidentiels en ville aboutit ainsi à la mise en place de constellations complexes d'individus et de cohabitation des générations. On enregistre ainsi des ménages avec seulement la génération des "parents" ; des ménages avec "grands-parents et parents", des ménages avec "parents et enfants"; des ménages avec "parents et petits enfants" ; des ménages avec "grands-parents, parents et enfants"; des ménages avec "grandsparents, parents et petits enfants"; des ménages avec "parents, enfants et petits enfants"; des ménages avec "grands-parents, parents, enfants et petits enfants". De façon générale, on note une primauté des ménages urbains à deux générations, composés essentiellement des parents et des enfants, suivis de la combinaison "parents, enfants et petits enfants". Les modèles urbains avec "grandsparents, parents et enfants" sont en troisième position, tandis que les ménages enregistrant la présence de toutes les générations en leur sein représentent environ $2 \%$ des structures de ménages.

Concernant la présence de la génération des grands-parents en milieu urbain, des fluctuations à la baisse ont été observées entre 1993 et 2010. Cette période correspond à une difficile période sur le plan socioéconomique, notamment pour les centres urbains au Burkina Faso. En effet, entre le début des années 1990 et le début des années 2000, le Burkina Faso, tout comme d'autres pays africains, a vécu successivement les conséquences négatives des programmes d'ajustements structurels, ainsi que les difficultés financières consécutives à la dévaluation de $50 \%$ de la parité du Franc CFA. Le milieu urbain caractérisé par une économie mieux structurée aurait été plus affecté par ces mesures économiques comparativement au milieu rural caractérisé par une économie informelle de subsistance (Ela, 1998). Ce qui aurait pour conséquence d'avoir éprouvé l'élan de solidarité résidentielle dans les villes (Wakam et al., 1998).

Dans leur processus d'extension à d'autres membres de la parentèle, les ménages urbains abritent de façon préférentielle beaucoup plus de personnes de la génération des petits enfants que de celle des grands-parents. La coexistence simultanée en ville de la génération des parents avec celle de leurs enfants et des enfants de ces derniers peut être interprétée à partir de plusieurs hypothèses. Premièrement, avec la crise socioéconomique et les difficultés de transition à l'âge adulte, les jeunes auraient du mal à accéder à l'autonomie financière et résidentielle. Aussi, resteraient-ils le plus longtemps sous la tutelle parentale avec leurs épouses et leurs enfants. Dans le sens de la réciprocité du contrat intergénérationnel, une deuxième explication serait qu'en l'absence d'un système de sécurité sociale moderne performant, les enfants constitueraient l'assurance retraite des parents qu'ils recueillent au sein de leurs ménages durant leurs vieux jours. L'élan de solidarité familial conduirait enfin les enfants socialement et économiquement autonomes à demeurer avec leurs propres familles (épouses et enfants) dans la grande famille paternelle en y assumant la responsabilité de chef, eu égard à l'incapacité de leurs pères à continuer d'assurer cette responsabilité du fait du poids de la vieillesse.

Par ailleurs, il faut retenir que les générations des grands-parents sont davantage présentes dans les ménages dont un homme en est le chef. A contrario, ce sont dans les ménages dont les femmes ont la responsabilité que l'extension vers les descendants (enfants et petits enfants) est la plus importante. Ce qui laisse entrevoir une sorte de division sociale des responsabilités de solidarité envers la parentèle étendue.

Les facteurs de modernisation, à savoir l'instruction du chef de ménage et le niveau de vie du ménage, ont des influences mitigées sur la présence dans le ménage des générations des grands-parents, des enfants et des petits enfants. Tandis que de façon globale on note une relation négative entre l'instruction du chef de ménage et la présence dans le ménage des différentes générations, l'amélioration du niveau de vie semble accroître la présence des générations des enfants et des petits enfants en milieu urbain notamment. Par contre, les ménages urbains avec un niveau de vie élevé seraient moins disposés à l'accueil des grandsparents en leur sein.

Q uel que soit le milieu de résidence, on note que la présence d'une génération quelconque influence négativement la présence d'une autre. La situation s'apparenterait à une sorte de contingentement des générations de façon à réduire autant que possible leur nombre.

\section{Conclusion}

Face à l'hétérogénéité des situations familiales et résidentielles, avec d'un côté la primauté de la famille nucléaire sur la parentèle étendue et d'un autre la résistance de la logique de parenté à l'individualisme, il y a lieu en effet de se poser la question à savoir où va la famille urbaine africaine ? A 
cette question, on convient avec Ela (1992 : X) que "Sans reproduire nécessairement les modèles importés ni rejeter systématiquement les pratiques ancestrales, les 'bricolages' auxquels se livrent de nombreux acteurs mettent en œuvre les stratégies diverses par lesquelles s'invente une nouvelle manière de vivre en famille dans les structures du quotidien."

Les modèles familiaux dans les villes africaines et plus spécifiquement du Burkina Faso laissent ainsi transparaitre une dualité reposant sur une sorte de conciliation entre les traditions familiales de parenté étendue et l'adaptation aux nouvelles exigences de la modernisation se traduisant par une préférence voire une primauté de la famille restreinte. A coté des constellations familiales complexes héritées des traditions familiales, évoluent aussi des ménages restreints basés sur une logique plus individuelle ou de couple.

\section{Bibliographie}

Antoine, P, Bocquier, P, Fall, A.S, Guissé, Y.M. et $N$ anitélamio, J. 1995. Etude de l'insertion urbaine à Dakar. In Antoine P. et Diop A.B. (ed) : La ville à guichets fermés ? Itinéraires, réseaux et insertion urbaine. IFAN, ORSTOM ; Dakar, Paris : $13-25$.

Antoine, P. 2007. Introduction. In Antoine, P. (ed) : Les relations intergénérationnelles en Afrique : approche plurielle. CEPED, Paris : 9-17.

Baya, B. et Laliberté, D. 2008. "C rises des solidarités ou solidarités dans la crise ? U ne lecture à partir de l'analyse de l'évolution de la structure des ménages entre 1993 et 2003 à Ouagadougou, Burkina Faso". Etude de la Population Africaine/African Population Studies 23(2):273287.

Benga, N.A. 1998. "Si on pouvait se contenter de la petite famille, on vivrait bien": élite urbaine, crise et processus d'individualisation à Dakar, Sénégal. Louvain-la-N euve UCL : 18.

Bongaarts, J. 2010. The Causes of Educational Differences in Fertility in sub-Saharan Africa. Working paper $\mathrm{N}^{\circ}$ 20. Population Council, $\mathrm{N}$ ew York : 20.

Cordell, D. et Piché, V. 1997. Pour une histoire de la famille en Afrique. In Pilon, M, Locoh, T, Vignikin, $K$. et Vimard $P$. (ed): Ménages et familles en Afrique: Approches des dynamiques contemporaines. Les Etudes du CEPED, 15, CEPED, Paris : 55-74.

Diagne A. et Lessault D. 2007. Émancipation résidentielle différée et recomposition des dépendances intergénérationnelles à Dakar. Collections Regards sur, CEPED, Paris : 41.
Ela, J-M. 1997. Préface. In Pilon, M, Locoh, T, Vignikin, K. et Vimard, P. (ed): Ménages et familles en Afrique: Approches des dynamiques contemporaines. Les Etudes du CEPED $\mathrm{N}^{\circ} 15$, CEPED, Paris : VII-X.

Ela, J-M. 1998. Population, pauvreté et crises. In Gendreau, F. (ed): Crises, pauvreté et changements démographiques dans les pays $d u$ sud. ESTEM, Paris : 17-34.

Hugon, P. 2008. Variables démographiques et éducation en Afrique ou le mirage des 0 bjectifs du millénaire pour le développement. Mondes en développement, $142: 83-96$.

IN SD et ICF International. 2012. Enquête Démographique et de Santé et à Indicateurs Multiples du Burkina Faso 2010. Calverton, Maryland, USA : 501.

Kinda, F. 2006. Contrat entre les générations et sécurité sociale locale des personnes âgées en milieu rural burkinabé. Cahiers du CERLESHS, 25. PU O, O uagadougou : 85-104.

Locoh, T. 1988a. L'analyse comparative de la taille de la famille et de la structure des ménages. African population conference - Congrès africain de la population, UIESP, Dakar 1988, volume II. UIESP, Liège : 5.2.17-5.2.40.

Locoh, T. 1988b. L'évolution de la famille en Afrique. In Van De Walle, E, Sala-Diakandan, D.M.et Ohadike, P. (ed): L'état de la démographie africaine : 45-66.

Locoh, T. 1997. Structure familiales: L'apport de la démographie. In Pilon, M, Locoh T, Vignikin, $K$. et Vimard, P. (ed): Ménages et familles en Afrique: Approches des dynamiques contemporaines. Les Etudes du CEPED, 15, CEPED, Paris : 408.

Marie, A. 1997. Les structures familiales à l'épreuve de l'individualisation citadine. In Pilon, M, Locoh $T$, Vignikin $K$. et Vimard, P. (ed) : Ménages et familles en Afrique: Approches des dynamiques contemporaines. Les Etudes du CEPED, 15, CEPED, Paris. : 408.

Pilon, M. et Vignikin, K. 2006. Ménages et familles en Afrique Subsaharienne. Savoirs francophones, Editions des archives contemporaines. AUF, Paris : 131.

Roth, C. 2007. "Tu ne peux pas rejeter ton enfant". Contrat entre les générations, sécurité sociale et vieillisse en milieu urbain burkinabé. Cahiers D'études Africaines, 185. EHESS, Paris : 93-116.

Tabutin, D. et Bartiaux, F. 1986. Structure familiales et structures sociales dans le tiers monde. In AIDELF (ed): Les structures familiales 
d'aujourd'hui : démographie et évolution récente des comportements familiaux. AIDELF, Paris: 231-243.

Tuman, J.P, Ayoub, S. et Roth-Johnson, D. 2007. The Effects of Education on Fertility in Colombia and Peru : Implications for Health and Family Planning Policies. Global Health Governance, I, (2) : 13.

Vignikin, K. 2007. Famille et relations intergénérationnelles: Réflexions sur les évolutions encours en Afrique. In Antoine, P. (ed): Les relations intergénérationnelles en Afrique : approche plurielle. CEPED, Paris : 1929.
Vimard, P. et N'Cho, S. 1997. Evolution de la structure des ménages et différenciation des modes familiaux en Côte d'Ivoire 1975 : 1993. In Pilon, M, Locoh, T, Vignikin, K. et Vimard, P. (ed) : Ménages et familles en Afrique: Approches des dynamiques contemporaines.. Les Etudes du CEPED, 15, CEPED, Paris : 101-123.

Wakam, J, Rwenge, M. et Kuépié, M. 1998. Pauvreté et structures familiales dans trois métropoles africaines : Yaoundé, Abidjan et Dakar. In Gendreau, F. (ed): Crises, pauvreté et changements démographiques dans les pays du sud. ESTEM, Paris : 167-182. 